Agrarpolitik

\section{Interessenausgleich erforderlich}

Lange Jahre war die Demonstrationsbereitschaft in der deutschen Landwirtschaft sehr überschaubar: Gab es in den 1960er Jahren noch vehemente Proteste sogar gegen akademisch verortete Empfehlungen zur Agrarpolitik (man denke an das „Professorengutachten“ 1962, in dem eine Senkung der politisch fixierten Agrarpreise mit flankierenden Maßnahmen vorgeschlagen wurde), führten die tiefgreifenden Reformen der Agrarpolitik, die mit den Namen der EU-Agrarkommissare MacSharry und Fischler verbunden sind, kaum dazu, dass Landwirte für die breite Öffentlichkeit sichtbar demonstriert haben. Nun hat sich diese Lage wieder verändert. Erste Anzeichen für eine gestiegene Demonstrationsbereitschaft der Landwirte waren bei der Internationalen Grünen Woche in den vergangenen Jahren zu beobachten, bei der quasi als Gegenpol zu den „Wir haben es satt"-Demonstrationen aus dem Spektrum der Nicht-Regierungsorganisationen die „Wir machen Euch satt"-Initiative regelmäßig auch in den Medien beachtet wird. Hier findet sich schon ein deutlicher Unterschied zu Bauerndemonstrationen der Vergangenheit: Nicht mehr das Agrarpreisniveau oder die Einkommensstützung im Allgemeinen stehen im Fokus der Demonstrierenden, vielmehr soll informiert und gesellschaftliche Anerkennung vor allem für die Nahrungsmittelerzeugung der Landwirtschaft erreicht werden.

Im Jahr 2019 hat diese Entwicklung weiter an Dynamik gewonnen. Vor allem jenseits der klassischen Lobbyorganisationen hat sich mit "Land schafft Verbindung" eine Initiative etabliert, die weitgehend über soziale Medien selbst organisiert ist und sich als Graswurzelbewegung sieht. Der wichtigste Auslöser war die Verschärfung der Düngeverordnung, die von vielen Landwirten als massive Einschränkung ihrer unternehmerischen Freiheit wahrgenommen wurde. Den Beteiligten ging es aber nicht ausschließlich um die Düngeverordnung: die deutschen Landwirte, die heute gerade noch für $0,7 \%$ des Bruttoinlandsprodukts stehen, fühlen sich mit aus ihrer Sicht ungerechtfertigten gesellschaftlichen Erwartungen vor allem im Bereich Umweltschutz und Tierwohl konfrontiert. Schwerpunkt der Aktivitäten war daher stärker als zuvor das Streben nach gesellschaftlicher Anerkennung. Daraus entwickelten sich deutlich sichtbare Demonstrationen, bei denen viele Landwirte mobilisiert wurden, sodass die Traktoren in Großstädten dann auch teils den Verkehr lahmlegten. Daraufhin

(C) Der/die Autor(en) 2020. Open Access: Dieser Artikel wird unter der Creative Commons Namensnennung 4.0 International Lizenz (https:// creativecommons.org/licenses/by/4.0/deed.de) veröffentlicht.

Open Access wird durch die ZBW - Leibniz-Informationszentrum Wirtschaft gefördert. wurde ein erster Bauerngipfel Anfang Dezember 2019 im Bundeskanzleramt anberaumt, in der die Landwirtschaft sehr breit vertreten war (Medien berichteten von über 40 Verbänden). Hier wurde von Bundeskanzlerin Merkel und Bundeslandwirtschaftsministerin Klöckner zugesichert, die gesellschaftlichen Leistungen der Landwirtschaft stärker zu betonen und im Dialog zu bleiben.

Diese kaum greifbaren Ergebnisse haben wenig überraschend nicht zu einer Beruhigung der Situation geführt. Nach landesweit fortgesetzten Demonstrationen hat der Koalitionsausschuss am 30. Januar 2020 die von Markus Söder als "Bauernmilliarde“ titulierte Unterstützung beschlossen, die vor allem die Anpassung an die Düngerverordnung erleichtern soll. Die Reaktion der traditionellen Lobbyorganisationen war - vorhersehbar - positiv; nicht so die Reaktion der Graswurzelbewegung. Hier war von dem Versuch einer Ruhigstellung der Landwirte die Rede, und es wurde betont, dass gerade nicht die Forderung nach erhöhten Subventionen, sondern das Schaffen von verlässlichen Rahmenbedingungen und gesellschaftlicher Anerkennung im Mittelpunkt stehen sollten. Der die Agrarpolitik in den letzten Jahrzehnten so häufig prägende Ansatz, dass notwendige Reformen mit Mitteln des Steuerzahlers „erkauft" werden, um so die Versäumnisse der Vergangenheit zu übertünchen, scheint diesmal nicht aufzugehen.

Es scheint geboten, dass die Agrarpolitik eine stärker moderierende Position einnimmt. Der Brückenschlag zwischen einer Bevölkerung, die kaum persönliche Einblicke in die Wirtschaftsweise von Landwirten hat, und den Bauern, die sich häufig durch die jenseits der Nahrungsmittelproduktion positionierten gesellschaftlichen Erwartungen überfordert sieht, macht eine Agrarpolitik erforderlich, die sich nicht einer bestimmten Klientel, sondern stärker dem Interessenausgleich verpflichtet fühlt. Dieser Spagat ist gewiss nicht einfach und wird durch die heterogene Interessenlage auf der Ebene der europäischen Agrarpolitik noch schwieriger. Hier sollte die deutsche Agrarpolitik aber umso deutlicher dafür eintreten, dass der erhebliche Finanzaufwand, der im Rahmen der Gemeinsamen Agrarpolitik der EU betrieben wird, endlich zielgerichtet für den gesellschaftlich gewünschten Umbau und für angemessene wirtschaftliche Rahmenbedingungen der Landwirtschaft verwendet wird. Das EU-Agrarbudget von gut $55 \mathrm{Mrd}$. Euro (2018) zu fast drei Vierteln auf Direktzahlungen zu verwenden, die ursächlich immer noch auf die Absenkung von Garantiepreisen für Agrarprodukte in den 1990er Jahren zurückgehen und zu großen Teilen zu einer Steigerung der Pachtpreise führen, kann kein sinnvoller Ansatz für eine zukünftige Agrarpolitik sein.

Bernhard Brümmer Georg-August-Universität Göttingen bbruemm@gwdg.de 